\title{
Effect of Surface Pressure Integration Methodology on Launch Vehicle Buffet Forcing Functions
}

\author{
Martin K. Sekula, ${ }^{*}$ David J. Piatak, ${ }^{\dagger}$ and Russ D. Rausch ${ }^{\ddagger}$ \\ NASA Langley Research Center \\ Hampton, VA 23681
}

\begin{abstract}
The 2014 test of the Space Launch System (SLS) Rigid Buffet Model conducted at the NASA Langley Transonic Dynamics Tunnel employed an extremely high number of unsteady pressure transducers. The high channel count provided an opportunity to examine the effect of transducer placement on the resulting buffetforcing functions (BFFs). Rings of transducers on the forward half of the model were employed to simulate a single-body vehicle. The impact of transducer density, circumferential distribution, and loss of a single transducer on the resulting BFFs were examined Rings of transducers on the aft half of the SLS model were employed to examine the effect of transducer density and circumferential distribution on BFFs for a multibody configuration. Transducer placement considerations with respect to model size, facility infrastructure, and data acquisition system capabilities, which affect the integration process, are also discussed.
\end{abstract}

\section{Notice to Readers}

The predicted performance and certain other features and characteristics of the Space Launch System vehicle are defined by the U.S. Government to be Sensitive But Unclassified (SBU). Therefore, values in plots and figures have been either removed or normalized to arbitrary values.

\section{Background}

$\mathrm{T}$ HE transonic buffet environment is a major contributor to the overall assent loads encountered by a launch vehicle. These buffet environments are typically determined through wind tunnel testing of rigid, subscale models. These models are equipped with hundreds of miniature unsteady pressure transducers embedded in their skins to measure the surface pressure environment. The products of these wind-tunnel tests are databases of time-correlated pressure time histories that are then employed in the development of buffet forcing functions (BFFs). These forcing functions are developed through a multistep process where some of these steps require the use of engineering judgment to make key decisions in the analysis. These decisions include surface pressure integration methodology, selection of similar aerodynamic regions for coherence analysis, and the selection of the frequency range of interest. Depending on choic es made, these decisions can affect the magnitude of the resulting BFFs, their frequency content, or both.

This w ork examines the impact of the integration process on the buffet forcing functions. There are two sets of decisions that are guided by engineering judgment that have a direct impact on the integration process. The first set is the selection of the pressure transducer locations on the wind-tunnel model; the second set is the selection of the surface integration boundaries associated with each transducer. These decisions begin during the model design process, and are discussed below in general terms, followed by a systematic analysis of how transducer location and integration boundaries can affect the resulting BFFs.

\footnotetext{
${ }^{*}$ Research Aerospace Engineer, Aeroelasticity Branch, martin.k.sekula@nasa.gov, Senior Member AIAA.

${ }^{\dagger}$ Research Aerospace Engineer, Aeroelasticity Branch, david.j.piatak@nasa.gov, Member AIAA.

‡Assistant Head, Aeroelasticity Branch, russ.d.rausch@nasa.gov, Associate Fellow AIAA.
} 


\section{A. Model Design Considerations}

The distribution of unsteady pressure transducers on the surface of a wind-tunnel model is typically guided by preliminary computational fluid dynamics (CFD) results and engineering judgment. Furthermore, the decisions on the number of transducers and their placement are subject to constraints such as model size and location accessibility as well as data acquisition system (DAS) and test facility infrastructure limitations. During model design, the following need to be considered when determining the number of transducers:

1. Model size - Larger models provide a larger interior volume that simplifies signal wiring, power distribution and signal amplification - if required. Also, larger models improve access in areas of the model where outer mold line (OML) changes create sharp angles and may cause interference between installed transducers, thereby limiting the transducer population density in regions where they may be most needed. Model size also affects the required sampling rate necessary to measure the required fult scale frequency range.

2. DAS channel count and data throughput limits - Higher channel counts can limit the sampling rate for acquiring the pressure time histories. Typically, unsteady pressure fluctuations below full-scale frequencies of 50 or $60 \mathrm{~Hz}$ are considered to be in the buffet frequency range. Therefore, a sufficientlyhigh sampling rate must be set to properly resolve unsteady pressures within the model-scale equivalent frequency range. It should be noted that the model-scale frequency range, and therefore sampling rate, are affected by the model size.

3. Facility infrastructure - Typically, rigid buffet models (RBMs) are sting-mounted models and some facilities may not have all DAS data channels wired to the sting support. Furthermore, the sting itself may further limit the allowable transducer count by the size of the bore in the sting and whether the ability exists to run the wiring external to the sting. Initial series of RBM tests at the NASA Langley Transonic Dynamics Tunnel were limited to 256 transducers (unsteady pressure transducers and accelerators) due to limitations of the DAS. As the tests grew in complexity, the DAS was updated to increase the channel count to 360 channels and currently it stands at 512 channels. This high channel count pushed the limits of the facility infrastructure, requiring creative solutions for transmitting transducer signal from the model to the DAS (such as repurposing wiring such as power for individual sensors, etc.,) to increase the available channels.

For the purposes of developing buffet forcing functions, unsteady pressure transducers are usually located in a series of rings at various longitudinal stations on the model. The locations of these rings are typically based on preliminary steady-state CFD solutions, where areas of high gradients in surface pressures are often most crucial for buffet-type aerodynamic phenomena. The exact location of transducer rings must consider the transducer count and physical constraints of the model design. Furthermore, the limits on the number of transducers require that compromises are made weighing azimuthal fidelity number of transducers in any single ring - versus longitudinal fidelity - number of longitudinal stations where pressures are measured. These decisions must consider the integration assumptions such as integration boundaries and regions of highly coherent flow.

\section{B. Motivation}

During wind tunnel tests conducted at the NASA Langley Transonic Dynamics Tunnel in support of NASA's former Constellation program, Ares I-X and Ares I RBMs were designed with either 4 or 8 axisymmetric ally-spaced transducers at any instrumented longitudinal station. ${ }^{1-3}$ An attempt was made to apply a similar approach in transducer density during the design of the Space Launch System (SLS) RBM tested at the TDT during a 2012 wind tunnel entry. The multi-body nature of this launch vehicle configuration presented a situation where both longitudinal and azimuthal fidelity needs could not be satisfied with the available channel count. The solution employed to circumvent this problem was a nonaxisymmetric azimuthal transducer distribution in the multi-body section of the model. More transducers were located on one side of the model compared to the other, providing a higher azimuthal fidelity on that 
side of the model, with the expectation that the information garnered from the higher density side could be extrapolated and applied to the other side of the model.

The loads resulting from this integration process were found to be very sensitive to the integration boundaries and motivated a follow-on 2014 wind-tunnel entry of the SLS RBM, where the nonaxisymmetric rings of transducers were fully populated and further expanded to include 16 transducers per ring. This high transducer density provided an opportunity to examine how transducer distribution around the circumference of the model affects the amplitude and frequency content of buffet forcing functions.

\section{Analysis}

The present analysis is divided into two parts. First, an analysis of six 8-transducer rings located on the forward half of the SLS RBM will be presented, simulating conditions experienced by a single-body vehicle. The second half of the analysis will focus on the impact of the integration process on the line loads on the multi-body (aft) section of the SLS RBM where seven 16-transducer rings are located. A graphical representation of the SLS RBM is presented in Figure 1. This figure highlights the locations and station designation numbers of both the 8-transducer rings (highlighted in red) on the front half of the SLS-10005 configuration of the model and of the 16-transducers rings on the aft half of the model (highlighted in yellow). The 16-transducer rings are a combination of new rings added for the 2014 entry and transducers added to fully populate the non-axisymmetric rings from the 2012 entry.

The data set analyzed included pressure measurements from 7028 wind tunnel test conditions. These test conditions included combinations of Mach numbers ranging from 0.80 to 1.18, model pitch angles ranging from $-8^{\circ}$ to $8^{\circ}$, and roll angles ranging from $-180^{\circ}$ to $180^{\circ}$, including repeat conditions. There were several configuration changes during this test documented in Ref. 4. These configuration changes did not affect the basic geometry of the SLS Core but did affect the aerodynamic environment on the aft half of the vehicle through protuberance and booster nosecone changes.

\section{A. Pressure Integration}

The standard practice in integrating the measured pressures on the surface of the vehicle model is to assume that the pressures are constant across the area of integration associated with each transducer. The integration boundaries for each of these areas are assumed to be located at either the midpoint (longitudinal and azimuthal) between transducers, or at large changes in the vehicle OML along the longitudinal axis of the vehicle. The pressures are assumed to act normal to the model surface and the effect of protuberances on the integration process is ignored.

For the purposes of this analysis, only the integration around the circumference of the vehicle is considered. The load components along the longitudinal axis of the vehicle, which arise when integrating on non-cylindrical vehicle segments, are neglected, resulting in the following equation for the sectional (force-per-length) line loads.

$$
\mathbf{f}=\sum_{i=1}^{n}-P_{i} r\left[\left(\cos \theta_{i+1}-\cos \theta_{i}\right) \hat{\mathbf{j}}+\left(\sin \theta_{i+1}-\sin \theta_{i}\right) \hat{\mathbf{k}}\right]
$$

Where $P_{i}$ is the measured pressure time history of the $i^{\text {th }}$ transducer, $r$ is the radius at the transducer station, $\theta_{i}$ and $\theta_{i+1}$ are the azimuth angles denoting the integration boundaries for the $i^{\text {th }}$ transducer, $\hat{\mathbf{j}}$ and $\hat{\mathbf{k}}$ are unit vectors in the lateral and vertical directions for the coordinate system of the SLS vehicle, and $\mathbf{f}$ is a vector containing the time histories of the orthogonal (lateral and vertical) components of the buffet forcing functions.

\section{B. Single-Body Integration}

An analysis was conducted to examine the effect of the number of transducers, their clocking, and removal of individual transducers on the integrated line loads of a single-body launch vehicle. The analysis considered six rings of 8 axisymmetric ally-spaced transducers, dispersed over the forward half of the SLS- 
10005 RBM (see Figure 1). The orthogonal sectional loads are developed by integrating the pressures around the vehicle circumference as described previously. The integration process was modified to permit the omission of transducers from the line load integration thereby simulating the following integration scenarios:

- A ring of 4-axisymmetrically-spaced transducers clocked with a 0-90 degree azimuth angle orientation

- A ring of 4-axisymmetric ally-spaced transducers clocked with a 45-135 degree azimuth angle orientation

- Both 4- and 8-transducer integrations with a single transducer removed to simulate the loss of a transducer due to a mechanical or electrical fault.

\section{Influence of Integration on Power Spectral Density Function}

The large dataset analyzed in this work makes it impractical to conduct detailed examinations of the BFF pow er spectral densities (PSDs) for each test condition, integration scheme, and transducer ring station. Therefore, a single flight condition $\left(\mathrm{M}=0.9, \alpha=0^{\circ}, \phi=0^{\circ}\right)$ is examined in detail. The PSDs of the vertical BFF components produced by 8- and 4- transducer integration for this sample flight condition are presented in Figure 2. The abscissa (frequency) scale for the PSDs at various stations is identical (and an approximate frequency range for buffet phenomena is provided), but the ordinate (spectral power) scale for the six stations varies from station to station in order to provide sufficient detail to discern the effect of integration on the PSDs. In each figure, the PSD of the 8-transducer BFF is provided in blue, the 4-transducers (0-90 degree orientation, referred to as $4_{1}$ ) BFF PSD is provided in red, and the 4-transducer (45-135 degree orientation, referred to as $4_{2}$ ) BFF PSD is provided in green. The 8-transducer-derived BFFs are considered the most accurate since they are developed from the highest-density measurements available at these stations; therefore, all other integration schemes will be compared to them. It should be noted that the PSD amplitudes are plotted on a log scale.

Four-transducer-based BFF PSDs at stations 12, 13, 15, and 19, Figures 2(b, c, d, and f), are conservative (higher amplitude) with respect to the 8-transducer PSD throughout the entire frequency range. At station 5, Figure 2a, the 4-transducer PSDs are also conservative (larger PSD amplitude than the 8-transducer baseline) within the typical buffet frequency range. But it should be noted that the PSD peak at frequencies just above the range typically associated with buffet phenomena is non-conservative for the $4_{1}$ integration BFF PSD, while the $4_{2}$ integration PSD is significantly over conservative. This disparity in conservatism is caused by an azimuthally-localized aerodynamic phenomena - the shed wake from four LAS nozzles located upstream of station 5. The difference in PSD magnitudes suggest that the transducers at the 45-135 degree clocking are located more directly in the LAS nozzle wake. Likew ise, at station 16, Figure 2e, the PSD of the BFF based on integration $4_{1}$ is non-conservative at low frequencies. This transducer-clockingdependency of the BFFs for station 16 indicates that the aerodynamic environment just aft of the large change in OML varies around the vehicle circumference.

\section{Root-Mean-Square Analysis}

To facilitate the analysis of a large dataset, the influence of the number and azimuthal distribution of transducers on the unsteady, integrated BFFs are quantified using a single metric, the root-mean-square (rms) levels of the unsteady component (mean removed) of the BFFs. Unless otherw ise stated, these rms levels will be normalized with respect to the 8 -transducer (baseline) BFF rms levels, ${ }^{\S}$ which are assumed to be the most-correct value as these are based on the highest transducer density available. The data is presented in a histogram format, where each histogram is based on approximately 84,000 BFFs (7028 wind tunnel test points, 6 stations, 2 BFF components per station). The legend in each histogram provides the

\footnotetext{
$\S$ For sake of brevity throughout this work, the discussion of rms levels implies the removal of the mean prior to its calculation.
} 
percentage of conservative cases (rms levels higher than ones produced by the baseline integration, i.e. greater than 1) for the integration scheme examined.

Histograms of the normalized rms levels for the 4-transducer integrated BFFs are presented in Figure 3. The data presented in blue corresponds to the integration of transducers situated in the 0-90 degree orientation, referred to as integration $4_{1}$, while the normalized rms levels for integration of transducers in the 45-135 degree orientation, integration 42 , is presented in red. For both integrations, the majority (95 percent) of the cases examined are conservative, meaning that the rms levels are increased compared to the baseline, 8-transducer integration.

Examining the data sorted by individual transducer stations provides further insight into the effect of transducers distribution on the BFF unsteadiness. The rms ratios of BFFs for both integrations, $4_{1}$ and $4_{2}$, are presented in Figure 4. While the reduced-transducer integration at all six stations produces mainly conservative results -90.4, 99.9, 99.6, 99.5, 90.1, and 94.9 percent of cases are conservative for stations 5, $12,13,15,16$, and 19 , respectively - three of the stations, stations 5,16 , and 19 , exhibit a lower percentage of conservative cases relative to the other stations. Station 5 is affected by localized shed wake from the LAS nozzles. Station 16 is just downstream of the expansion corner on the spacecraft adapter and therefore subject to a significantly unsteady aerodynamic environment characterized by detached flow and expansion shocks. These aerodynamic phenomena can be circumferentially localized and are affected by flight conditions (Mach number and vehicle attitude) and therefore may require a higher transducer density to properly resolve. Station 19 is another example of a localized aerodynamic phenomena affecting the BFFs. Examining integrations $4_{1}$ and $4_{2}$ individually (not presented) indicates that integration $4_{2}$ is more prone to underpredicting the fluctuation levels in the integrated BFFs - 97.8 versus 92.0 percent, respectively. This difference may be associated with transducers nearest to the boosters ( 90 and $270^{\circ}$ azimuth) measuring aerodynamic phenomena related the proximity to the boosters. Based on these observations and previous discussion of sample PSDs, it can be concluded that when determining transducer distribution on a buffet model, a higher number of transducers should be located at stations just down stream of large OML changes, large protuberances positioned at multiple locations around the circumference of the vehicle, and proximity to attached boosters.

The impact of the loss of a single transducer on the BFFs is examined in Figures 5 and 6. This analysis was conducted by systematically removing a single transducer from the integration to simulate its loss, adjusting the integration boundaries accordingly, and reintegrating the BFFs. A histogram of the ratio of rms values for 7-transducer integration BFFs to 8-transducer integration BFF is presented in Figure 5. Overall, the 7-transducer integration is conservative 92.8 percent of the time compared to 8-transducer integration. The histogram depicting the rms levels for BFFs based on a 3-transducer integration normalized with respect to the rms levels for BFFs based on four transducers is presented in Figure 6. This figure presents the combined data for the loss of a single transducers in both integration $4_{1}$ and integration $4_{2}$. The three transducer integration is conservative 88.3 percent of the time.

\section{Multi-body Integration}

The SLS RBM included seven longitudinal stations where 16 transducers were axi-symmetrically distributed around the circumference of the SLS Core. These stations are highlighted in yellow in Figure 1. The distribution of these transducers around the circumference of the SLS Core, provided in Figure 7, is identical for each of the seven stations. This figure also indic ates the locations of the two boosters relative to these transducers. The boosters play an important role in the analysis presented in this work, since the aerodynamic interaction between them, the SLS Core, and the attachment hardware created a significant buffeting phenomenon discussed in References 5 and 6.

The 16-transducer integration, having the highest transducer density, is considered the most accurate integration for the purposes of this work; therefore all other transducer integration scenarios will be compared to it. Both axisymmetric and non-axisymmetric transducer distributions are examined. The axisymmetric transducer distributions, presented in Figure 8, are as follows:

- 8-transducer distribution clocked at $0^{\circ}-45^{\circ}$, Figure 8 a, referred to as distribution 8 . 
- 8-transducer distribution clocked at $22.5^{\circ}-67.5^{\circ}$, Figure $8 b$, referred to as distribution 82 .

- 4-transducer distribution clocked at $0^{\circ}-90^{\circ}$, Figure 8c, referred to as distribution $4_{1}$.

- 4-transducer distribution clocked at $45^{\circ}-135^{\circ}$, Figure 8d, referred to as distribution 42 .

The non-axisymmetric transducer distributions all consisted of six transducers distributed around the circumference of the SLS Core at either 45 or 90 degree intervals. The following five non-axisymmetric transducer distributions, presented in Figure 9, are examined:

- Transducer distribution utilized during the 2012 wind tunnel entry, Figure 9a, referred to as distribution 61 .

- Transducer distributions $62-64$, presented Figures 9(b-d), employ the same transducer distribution as $6_{1}$ but rotated by $180^{\circ}, 90^{\circ}$, and $270^{\circ}$, respectively.

- $\quad$ Transducer distribution symmetric about the $0^{\circ}-180^{\circ}$ axis, Figure $9 e$, referred to as distribution $6_{5}$.

\section{Influence of Integration on Power Spectral Density Function}

Transducer density and their azimuthal loc ation on a multi-body vehicle can affect the frequency content of BFFs. The effect of various transducer distributions on BFF PSDs at a sample flight condition (M=0.9, $\alpha=0^{\circ}, \phi=0^{\circ}$ ) and a sample transducer station (Station 25) is presented in Figures 10 and 11. PSDs in the vertical and lateral directions for several transducer distributions are compared to PSDs derived from a 16transducer integration. In these figures, the two components of the PSDs are plotted on the same log scale for ease of comparison.

In previous work, an aerodynamic phenomenon associated with the forward attachment of the boosters to the SLS Core was identified. ${ }^{5,6}$ This phenomenon occurs in the vicinity of each booster and is comprised of two oscillating shocks, one on either side of the booster, oscillating $180^{\circ}$ out of phase with respect to each other as they respond to the shed wake from the forward attachment hardware. This interaction produces a large, highly-coherent oscillating flow structure affecting large areas of the SLS Core. This phenomenon manifests itself as two peaks in the PSD of the lateral component of the BFF, Figure 10a. The lower frequency peak is caused by the flow oscillation on either side of the boosters, while the higher frequency peak, at twice the frequency of the lower one, is produced by pressures measured directly in the gap between the boosters and the Core.

The PSDs of BFFs developed using various axisymmetric transducer distributions are presented Figure 10. The PSDs of the 8- and 4-transducer integrations highlight the effect of transducer distribution on the frequency content of the integrated BFFs. The PSD of lateral BFF obtained using integration $8_{1}$ overpredicts the high-frequency peak while correctly predicting the amplitude of the low-frequency peak. Conversely, the $8_{2}$ integration underpredicts the high-frequency peak since it doesn't include the measurements from the transducers located in the gap between the boosters and Core. Yet, this same integration method correctly predicts the low-frequency peak amplitude due to the larger integration areas associated with the transducers located on either side of the boosters $\left(67.5^{\circ}\right.$ and $112.5^{\circ}$ for the right SRB and $247.5^{\circ}$ and $292.5^{\circ}$ for the left booster). At frequencies away from these two peaks, integrations $8_{1}$ and $8_{2}$ are both generally conservative with respect to the 16 -transducer integration.

The PSD of the vertical component of the BFF developed using the 16-transducer integration (Figure 10b, blue line) exhibits only the low-frequency peak, since the transducers located in the gap between the Core and boosters $\left(90^{\circ}\right.$ and $270^{\circ}$ azimuth) do not contribute to the vertical BFF component. The BFF based on integration $8_{1}$ underpredicts this low frequency peak while the $8_{2}$ integration overpredicts it. These differences in PSDs are directly due to the locations of the transducers employed in the integration process and the larger areas of integration resulting from the larger separation distance between transducers compared to the 16-transducer integration.

The 4-transducer integrations exacerbated the transducer-clocking-related trends noted in the 8transducer integrations, Figure 10, since the circumferential integration lengths for each transducer increase due to the larger separation between transducers (recall transducer distribution presented in Figure 8). In 
fact, integration $4_{1}$ produces an approximately order-of-magnitude increase in the amplitude of the highfrequency peak compared to the 16-transducer integration, while in general, overpredicting the lateral BFF PSDs at all frequencies. At low frequencies which are more likely to contribute to the buffet loads, the $4_{1}$ integration doubles the PSD levels relative to the 16-transducer integration. This PSD overprediction is also true for the vertical BFF, with the exception of frequencies in the vicinity of the low-frequency peak (see Figure 10b, green line). This peak is not well defined in the integration $4_{1}$ PSD, but it should be noted that the lateral component is the dominant component of this BFF. Integration $4_{2}$ signific antly underpredicts the lateral BFF PSD throughout the entire frequency range of interest (Figure 10a, black line). The two peaks found in lateral BFF PSD of the 16-transducer integration are much diminished in integration $4_{2} \mathrm{BFF}$, as well as the lower-frequency peak found in the vertical BFF PSD. These observations indicate that the aerodynamic phenomenon which produces these peaks is mostly dissipated at Core locations $\pm 45^{\circ}$ away from the azimuthal positions of the boosters.

The effects of non-axisymmetric transducer distributions on the PSDs of the lateral and vertical BFF components are presented in Figure 11. The lateral BFF PSDs for integrations $6_{1}, 6_{2}, 6_{3}$, and $6_{4}$ all exhibit similar tends compared to the 16-transducer integration. These four integration schemes all overpredict the amplitude of the PSD throughout most of the frequency range on interest, culminating with a 5- to 6-fold overprediction of the high-frequency peak. BFF based on integration $6_{5}$ exhibits similar trends, although the lateral PSD high-frequency peak amplitude overprediction is only 2.5 times greater than baseline. This same integration approach underpredicts the vertical BFF PSD. This underprediction is associated with the exclusion of transducers at $0^{\circ}$ and $180^{\circ}$ azimuth from integration 65 , which measure a highly unsteady pressure environment due to their proximity to the feedlines.

The PSDs of the vertical BFF components for integrations $6_{1}, 6_{2}, 6_{3}$, and $6_{4}$, Figure $11 \mathrm{~b}$, overpredict the PSD amplitude at most frequencies, except in the vicinity of the low-frequency peak. All four of these pressure integration schemes underpredict this peak. But a more important observation is that integrations $6_{1}$ and $6_{2}$ both result in a large high-frequency peak not present in the 16-transducer integration. This false peak is a result of the asymmetric integration boundaries for the transducers at 90 and $270^{\circ}$ azimuth. The asymmetry in the integration boundaries is due to the assumption that each transducer's area of integration extends to the midpoint between it and the adjacent transducers. A graphical representation of this asymmetry for integration $6_{1}$ is presented in Figure 12 a. Since transducers at $90^{\circ}$ and $270^{\circ}$ azimuth measure the largest unsteady pressures caused by the interaction between the Core and the boosters, reducing the size of their integration area and making the location of the azimuthal integration boundaries symmetric about the transducer (Figure 12b) eliminates the false peak in the vertical BFF and can reduce the amplitude of the high-frequency peak in the lateral BFF. These adjusted integration boundaries are denoted by an "a” superscript in the integration naming convention.

It should be noted that the false high-frequency peak observed in the vertical BFF component of integrations $6_{1}$ and $6_{2}$ is also a function of longitudinal station. As stated earlier, this peak is created by a combination of shed wake from the booster forward attachment and asymmetric integration boundaries. Comparing the vertical BFF component PSDs based on the 16-transducer integration and integration $6{ }_{1}$, Figure 13, indicates that this false peak has disappeared in integration $6{ }_{1}$ PSDs for stations 29 through 31, Figures 13(e-g). This observation indicates that the wake from the booster attachment has significantly dissipated by station 29 .

The PSDs resulting from the integration boundary adjustment are presented in Figure 14. By adjusting the azimuthal integration boundaries for transducers located at $90^{\circ}$ and $270^{\circ}$ azimuth, the high-frequency peak in the lateral BFF PSD has been reduced by more than 50 percent (compare Figure 14 and Figure 11, same ordinate scale). The PSDs for the vertical BFFs indic ate that the false peak produced by integrations $6_{1}$ and $6_{2}$ are eliminated, see Figure $14 \mathrm{~b}$. In general, all the adjusted integrations $\left(61^{\mathrm{a}}, 6_{2}{ }^{\mathrm{a}}, 6^{\mathrm{a}}\right.$, and $\left.64^{\mathrm{a}}\right)$ still overpredict the PSD amplitude compared to the 16-transducer integration throughout the entire frequency range of interest but simultaneously underpredict the low frequency peak in the vertical BFF component. 


\section{Root-Mean-Square Analysis}

The effect of the number and azimuthal distribution of transducers on root-mean-square levels of the unsteady component (mean removed) of the BFFs is examined. Unless otherwise stated, these rms levels will be normalized with respect to the 16-transducer BFF rms levels, which are assumed to be the most representative of the actual buffet loads.

The data for the normalized-rms levels for the 8-transducer integrated BFFs are presented in a histogram format in Figure 15. Each of the histograms provided in Figure 15 is produced by examining approximately 100,000 BFFs (7028 wind tunnel test points, 7 stations, 2 BFF components per station). Unlike the results presented in the single-body analysis, the effect of transducer clocking has a profound effect on the resulting BFFs. The interactional aerodynamics between the multiple bodies (Core and boosters) has a substantial localized effect - pressure fluctuations measured on the Core increase with proximity to the boosters where the largest pressures occur in the gaps between the boosters and Core. ${ }^{5,6}$ Therefore, missing this peak fluctuation can underpredict the BFF fluctuation levels. This observation is noted by examining the percentage of BFFs whose rms levels are conservative (greater than 1) with respect to their 16-transducerbased counterparts. Integration scheme $8_{1}$, Figure $15 \mathrm{a}$, which includes the transducers located in the gaps between the Core and boosters, is conservative for 93.8 percent of the cases, while integration 8 , Figure $15 \mathrm{~b}$, is conservative only 53.2 percent of the time. It should be noted that the local aerodynamic phenomena caused by feedlines also contributes to the difference in BFF rms levels.

This clocking-based disparity is exacerbated by employing a 4-transducer integration. Integration scheme $4_{1}$, presented in Figure 16a, is conservative for 99.9 percent of the cases. Furthermore, the rms levels are significantly higher than for integration scheme 81 , the most similar 8-transducer integration (compare Figure 16a to Figure 15a). This increase in rms levels is caused by an increase in the integration area associated with each transducer because the number of transducers has been reduced. Integration scheme $4_{2}$ sees an opposite effect. The transducers employed in this pressure integration are located half way between the boosters and the previously mentioned feedlines. Therefore, at each transducer station, they are located in one of the more benign aerodynamic environments. This transducer location results in integration $4_{2}$ being conservative for only 39.8 percent of the cases examined.

Further insight into the effect of integrations $4_{1}$ and $4_{2}$ on the BFF integration can be found by examining how the rate of conservative cases changes with vehicle station, Figure 17. Histograms for integrations $4_{1}$ are presented in blue while ones for integration $4_{2}$ are presented in red. The unsteady aerodynamic phenomenon which dominates the SLS Core aerodynamic environment in this region is associated with the forward attachment of the boosters to the SLS Core, and the largest unsteady pressure fluctuations are measured by the first transducer station downstream of, and directly behind, the booster attachment hardware.5,6 Accordingly, integration 42 exhibits the lowest rate of conservative cases at this station, see Figure $17 \mathrm{a}$ - station 25 . As the transducer rings are located further downstream of the attachment hardware, the rate of conservative cases for integration 42 steadily increases (except at station 27), reaching 68 percent at station 31, Figure 17g, which is located approximately half way between the attachment hardware and the aft end of the vehicle. It is conceivable that the rate of conservative BFFs may keep increasing for transducer stations further down the vehicle, but probably will never reach the rates of conservatism associated with integration $4_{1}$.

Histograms presenting normalized rms levels of BFFs derived with non-axisymmetric integrations $6_{1}$, $6_{2}, 6_{3}, 6_{4}$, and $6_{5}$ for all stations are presented in Figure 18. The histograms for the lateral (blue) and vertical (red) components of the BFFs for each integration are provided. Integrations $6_{1}$ through 64 - Figures 18 (ad) - all exhibit high rates of conservative cases compared to the 16-transducer-derived BFFs. Based on the rms levels, the vertical component of the BFFs exhibit much higher levels of conservatism (denoted by the histograms range on the abscissa) than the lateral component. Integration 65 results in significantly lower rates of conservatism than the other non-axisymmetric transducer distribution cases. The vertical component is conservative for less than 30 percent of the cases analyzed which indicates that the feedlines provide a signific ant contribution to the unsteady integrated loads in integration 81 (compare Figure 15a and Figure 18e). Further studies, such as CFD analysis, should be conducted to examine the azimuthal extent of the feedline-produced environment. The results of such a study could be used to adjust the 
integration boundaries for the transducers near the feedlines to properly represent the influence of the feedlines on the BFFs.

The integration boundaries of integrations $6_{1}$ through $6_{4}$ were adjusted to more correctly represent the extent of the area of the SLS Core influenced by the aerodynamic interaction with the boosters. Histograms indicating the impact of this integration adjustment on the rate of conservative cases is presented in Figure 19 , with the individual integrations now labeled with the superscript " "a" to denote the integration boundary adjustment. Overall, the integration adjustment has reduced the rate of conservative cases in the lateral components of BFFs based on integrations $6_{1}$ and $6_{2}$ from near 100 percent to 70 percent, compare Figures 19( $a$ and $b$ ) with 18( $a$ and $b$ ). At the same time, the level of conservatism of the vertical BFF component was increased by these integrations, with the average value of rms ratio for the conservative cases increasing from 2.13 and 2.02 to 2.57 and 2.59 for integrations 61 and 62 , respectively. The increase in the vertical BFF rms levels due to the integration adjustment suggests that even though the false peak noted in the sample PSDs (Figure 11b) w as eliminated, the broadband fluctuation levels have increased. This increase can be attributed to the larger integration areas associated with transducers located at $0^{\circ}$ and $180^{\circ}$ azimuth - transducers situated in the vicinity of the feedlines.

Integration boundary adjustments to integrations $6_{3}$ and $6_{4}$ did not significantly affect the high rate of conservatism noted in Figures 18(c and d), compare with Figures 19(c and d). Integration $63^{\mathrm{a}}$ results in an increase in the average lateral rms ratio from 1.25 to 1.69 compared to integration $6_{3}$, while the average vertical rms ratio decreases from 2.59 to 2.04. Integration $64^{\mathrm{a}}$ results in an increase in the average vertical rms ratio from 2.02 to 2.40 , while having a very limited effect on the lateral rms ratio.

\section{Buffet Frequency Range Analysis}

As mentioned previously, it would be impractical to perform a detailed examination of the PSD of each $\mathrm{BFF}$ in this study. Therefore, a metric is required to provide an overarching quantification on how each integration scheme affects the BFF frequency content within a specified frequency range of interest. The approach employed examines the change in the total energy in the BFF in the buffet frequency range compared to the baseline, 16-transducer integration. Mathematically, this metric was defined as the difference in the areas-under-the-curve between the reduced-transducer and 16-transducer PSDs, normalized with respect to the area-under-the-curve of the 16-transducer PSD. This metric is given as:

$$
\frac{\int_{f_{1}}^{f_{2}}\left(P S D_{n}(f)-P S D_{16}(f)\right) d f}{\int_{f_{1}}^{f_{2}} P S D_{16}(f) d f}
$$

where $f_{1}$ and $f_{2}$ define the frequency range for the integration, and the subscript on the function PSD indicates the number of transducers in the integration. Based on this metric, values greater than zero indicate that the BFF is more energetic (and therefore probably conservative) in the frequency range examined. This metric is related to the rms ratio metric previously used in this work, since the integral of the PSD function is equal to the square of the rms. Therefore, the rms ratio is a broadband integration while the current metric focuses on just the buffet frequencies.

It should be noted that buffet is a frequency dependent phenomenon where aerodynamic forces interact with the vehicle's structural dynamics. Therefore the authors acknowledge that to state that an increase in the overall energy content over a wide range of frequencies is considered conservative is an oversimplification of the problem. While overall energy content over a frequency range may increase due to a change in the integration process, if the energy is artificially reduced through the integration process at a critical frequency that excites the structure, the BFF may actually not be conservative. But for the sake of conciseness, an increase in overall energy content will be referred to as "conservative." A true measure of the conservatism of a BFF would require the use of a coupled loads analysis (CLA), which is beyond the scope of this study. Other metrics, such as changes in amplitudes or frequencies of PSD peaks, can also be used to better understand the influence of the integration process, but are also beyond the scope of this study. 
Histograms of the normalized difference in the energy content between the 8-transducer-based and 16transducer-based BFFs is presented in Figure 20. Two sets of histograms are presented in this figure, each examining the change in energy content of the BFFs in different frequency ranges: (a) the lower half of the buffet frequency range and (b) the upper half of the buffet frequency range. The first item of note is the contrast in the percentage of conservative cases for integrations $8_{1}$ and $8_{2}$ regardless of the frequency range selected (approximately 90\% vs. 50\%), presented in Figure 20 as blue and red histograms, respectively. Integration $8_{1}$ is twice as likely to produce conservative BFFs than integration $8_{2}$ due to the placement of the transducers, as discussed previously. A closer examination of the low and high buffet frequency energy content for integration $8_{1}$, Figures 20 ( $a$ and $b$ ), indicates that the rate of conservatism of the $8_{1}$ integration is somewhat reduced at higher frequencies, but at the same time, the high-frequency cases that see an increase in energy are conservative by a much greater margin, indicated in the histogram by a shift to the right in the distribution of the data.

The normalized difference in the energy content between the 4-transducer-based and 16-transducerbased BFFs is presented in Figure 21. Integration $4_{1}$ results are presented in blue while integration $4_{2}$ results are presented in red. The trends in the energy change are similar to those seen in the rms trends (Figures 15 and 16), where the larger azimuthal integration range combined with the clocking angle of the transducers either make the integrations more conservative (average increase in BFF energy for integration $4_{1}$ is significantly higher than for $8_{1}$ ) or less conservative (integration $4_{2}$ vs. $8_{1}$ ). Unlike the 8-transducer integration, the percentage of conservative cases for the high-frequency buffet range increased compared to the 16-transducer integration. In particular, integration $4_{1}$ was conservative for 96.2 percent of the cases in the low-frequency buffet range and 99.3 percent of the time in the high frequency buffet range.

The influence of non-axisymmetric transducer distribution on energy content of the BFF is presented in Figures 22 through 26. Integrations $6_{1}$ and $6_{2}$ both exhibit slightly higher rates of conservatism in the higher buffet frequency range than in the lower frequency range, approximately 96 percent vs. 99 percent, see Figures 22 and 23. Examining just the conservative cases $(\triangle \mathrm{PSD}>0)$, the average increase in BFF energy content for the low-buffet-frequency range (compared to the 16-transducer BFFs) is approximately 160 percent in the lateral component compared to approximately 250 percent increase in the vertical component. For the high-buffet-frequency range, these two integration schemes create an average increase in BFF energy - compared to the 16-transducer BFF - by approximately 215 and 465 percent respectively for the lateral and vertical BFF components. Integrations $6_{3}$ and $6_{4}$, Figures 24 and 25, exhibit similar trends to integrations $6_{1}$ and $6_{2}$ where the energy in the high-buffet-frequency range due to the integration of nonaxisymmetric transducer distribution has a higher rate of conservative cases compared to the low-frequency buffet range. Within the conservative cases, the high-buffet-frequency data indicates a higher level of conservatism with respect to the 16-transducer-derived BFF than the low-frequency data (right-ward shift of distribution in histogram).

Examining the change in energy for the buffet frequency range for BFFs based on integration $6_{5}$ compared to BFFs based on the 16-transducer integration is presented in Figure 26. For both the highbuffet-frequency and low-buffet-frequency signals, this integration scheme results in 60 to 67 percent of the cases being non-conservative in the vertical BFF component. The rate of conservatism in the lateral BFF component seems to be lower for the high-buffet-frequency range when compared to the low-buffetfrequency range, 85.8 versus 96.5 percent, see Figure 26.

The effect of adjusting the integration boundaries for integration schemes $6_{1}$ through 64 (i.e. $6_{1}{ }^{a}$ through $64^{a}$ ) are presented in Figures 27 through 34. For each integration scheme, two sets of histograms are presented. First, histograms for transducer stations 25 through 27 are discussed; which, based on the prior PSD discussion, are susceptible to a false high-frequency peak in the vertical BFF component PSD (see Figure 11); and second, histograms for stations 28 through 31, which did not exhibit the false PSD peak. The aerodynamic environment measured at stations 28 through 31 is sufficiently altered compared to stations 25 through 27 , such that integrations $6_{1}{ }^{\mathrm{a}}$ and $6_{2}{ }^{\mathrm{a}}$ result in signific antly different histograms depicting the effect of integration on the BFF energy content, compare Figure 27 to Figure 28 and Figure 29 to Figure 30. The differences are readily apparent for both low- and high-buffet-frequency ranges. For the transducer stations nearest to the booster attachment, Figures 27 and 29, these integration schemes produce a higher 
percentage of conservative results in the lateral BFF component when compared to stations further downstream, Figures 28 and 30. This trend holds true regardless of the frequency range selected. These figure also indicate that the choice of longitudinal station has a limited effect on the rate of conservatism in vertical BFF component in the buffet frequency range for BFFs based on integrations $6_{1}{ }^{\mathrm{a}}$ and $6_{2}{ }^{\mathrm{a}}$. For both integrations and low- and high-buffet frequency ranges the rate of conservative vertical BFFs with respect to the 16-transducer integration is near 100 percent. But it should be noted that BFFs for stations 28 through 31 are more conservative than BFFs for stations 25-27. The mean increase in the vertical BFF energy content in the low-buffet-frequency range is approximately 300 percent for stations 25-27, but it grows to 450 percent for stations 28-31. Similarly, for the high-buffet-frequency range, the mean increase in the vertical BFF energy is more than 500 percent for stations 25-27, and almost 800 percent for station 28-31. It should be noted that the magnitude of these values is somewhat deceiving, since the energy content of the BFFs drops significantly with increasing separation distance from the booster forward attachment. This trend is discernable in Figure 13, where the PSDs of the 16-transducer-derived BFF are plotted at various transducer stations.

Histograms for the change in BFF energy content in the buffet frequency range for integration $63^{\mathrm{a}}$ and $64^{\mathrm{a}}$ are presented in Figures 31 through 34. These figures indicate that these integration schemes produce conservative results for 92 to almost 100 percent of the cases examined, regardless of buffet frequency range or longitudinal stations chosen. Both the vertical and lateral BFF components for integration $6_{3}{ }^{\mathrm{a}}$ and $64^{\mathrm{a}}$ exhibit a trend where the mean increase in the energy content of the BFFs is larger for stations located further from the booster attachment - a trend also identified in the vertical BFF components for integrations $61^{\mathrm{a}}$ and $62^{\mathrm{a}}$.

\section{Conclusions}

Data from a Space Launch System (SLS) Rigid Buffet Model wind-tunnel test was examined to determine how various pressure integration schemes affect the resulting buffet forcing functions (BFFs). Several highly-populated rings of transducers situated on the forward and aft half of the model were selected to conduct this analysis. Transducers on the forward half of the model were employed to examine a singlebody launch vehicle scenario, while transducers on the aft half of the model were utilized to examine a multi-body configuration. Root-mean-square levels of the fluctuating component of BFFs and PSDs of the BFFs integrated over a buffet frequency range were used as a metric to examine the impact of the various transducer integration schemes on the BFFs. Histograms were used to as a measure of conservatism for the integration schemes based on the lower-transducer-density. PSDs for a sample flight condition were also examined to provide further insight into the results of the integration process.

Based on the results of these analyses, the following conclusions and observations were made:

- Higher azimuthal transducer densities should be utilized in areas where large OML changes occur as well as downstream of protuberances which azimuthally cover large portions of the circumference, such as LAS nozzles. These areas may experience azimuthally-localized aerodynamic phenomena and low azimuthal transducer density can either substantially overpredict or underpredict the BFF depending on transducer location.

- For a single-body vehicle, lower azimuthal transducer density tends to increase the rms levels of the BFFs, unless a localized aerodynamic phenomenon is present. For multi-body vehicles, this trend does not hold true since transducer location plays an important role in dictating the fluctuation levels in the BFFs.

- Non-axisymmetric transducer distributions in the multi-body configuration can produce peaks in the BFF PSDs that do not exist in BFFs developed using an axisymmetric transducer distribution. Adjustments in the azimuthal integration boundaries can be made to remove these false peaks, but can create complications, such as lower rates of conservatism in certain cases or overprediction of BFF levels of fluctuation for other cases. Based on these findings, non-axisymmetric transducer distributions should be avoided, if possible. 


\section{References}

${ }^{1}$ Sekula, M. K., et al., "NASA Test Summary Document for the 3.5 Percent Ares I-X Rigid Buffet Model; Transonic Dynamic Test \#599; November-December 2007,” NASA TP-2015-218703, March 2015.

${ }^{2}$ Piatak, D. J., et al., "Test Summary Document for the 3.5 Percent Ares I Rigid Buffet Model; Transonic Dynamics Tunnel Test 605; Conducted from November to December 2008,” ARES-AE-TA-0012, March 2009.

3Piatak, D. J., Sekula, M. K., and Rausch, R. D., "Ares Launch Vehicle Transonic Buffet Testing and Analysis Techniques.” Journal of Spacecraft and Rockets, Vol. 49. No 5. September-October 2012. ${ }^{4}$ Piatak, D. J., Sekula, M. K., Rausch, R. D., Florance, J. R., and Ivanco, T. G., "Overview of the Space Launch System Transonic Buffet Environment Test Program," Proceedings of the $53^{\text {rd }}$ AIAA Aerospace Sciences Meeting, AIAA 2015-0557, January 5-9, 2015.

${ }^{5}$ Sekula, M. K., Piatak, D. J., Rausch, R. D., "Initial Assessment of Space Launch System Transonic Unsteady Pressure Environment," Proceedings of the 53 ${ }^{\text {rd }}$ AIAA Aerospace Sciences Meeting, AIAA 2015-0558, January 5-9, 2015.

${ }^{6}$ Brauckmann, G. J., Streett, C., Kleb, W. L., Alter, S. J., Murphy, K. J., and Glass, C. E., “Computational and Experimental Unsteady Pressures for Alternate SLS Booster Nose Shapes," Proceedings of the 53"d AIAA Aerospace Sciences Meeting, AIAA 2015-0559, January 5-9, 2015. 


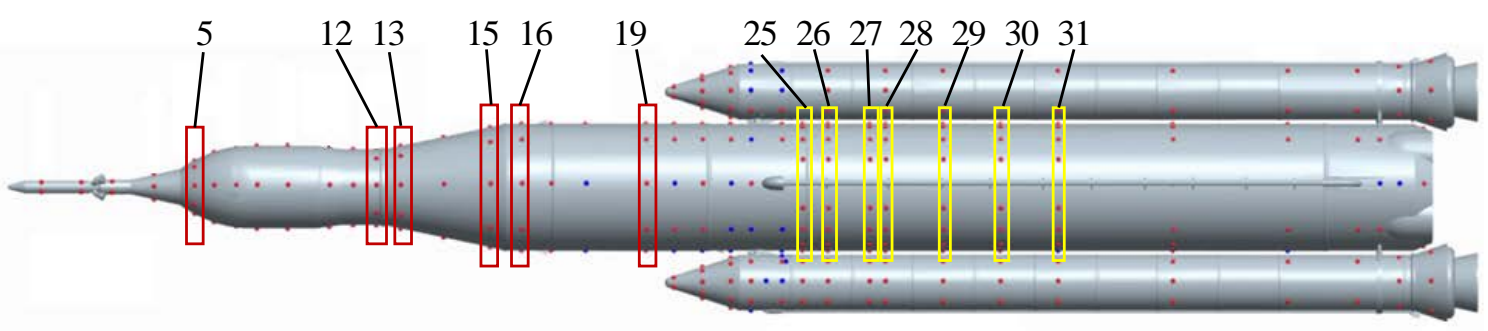

Figure 1. SLS-10005 RBM transducer layout.

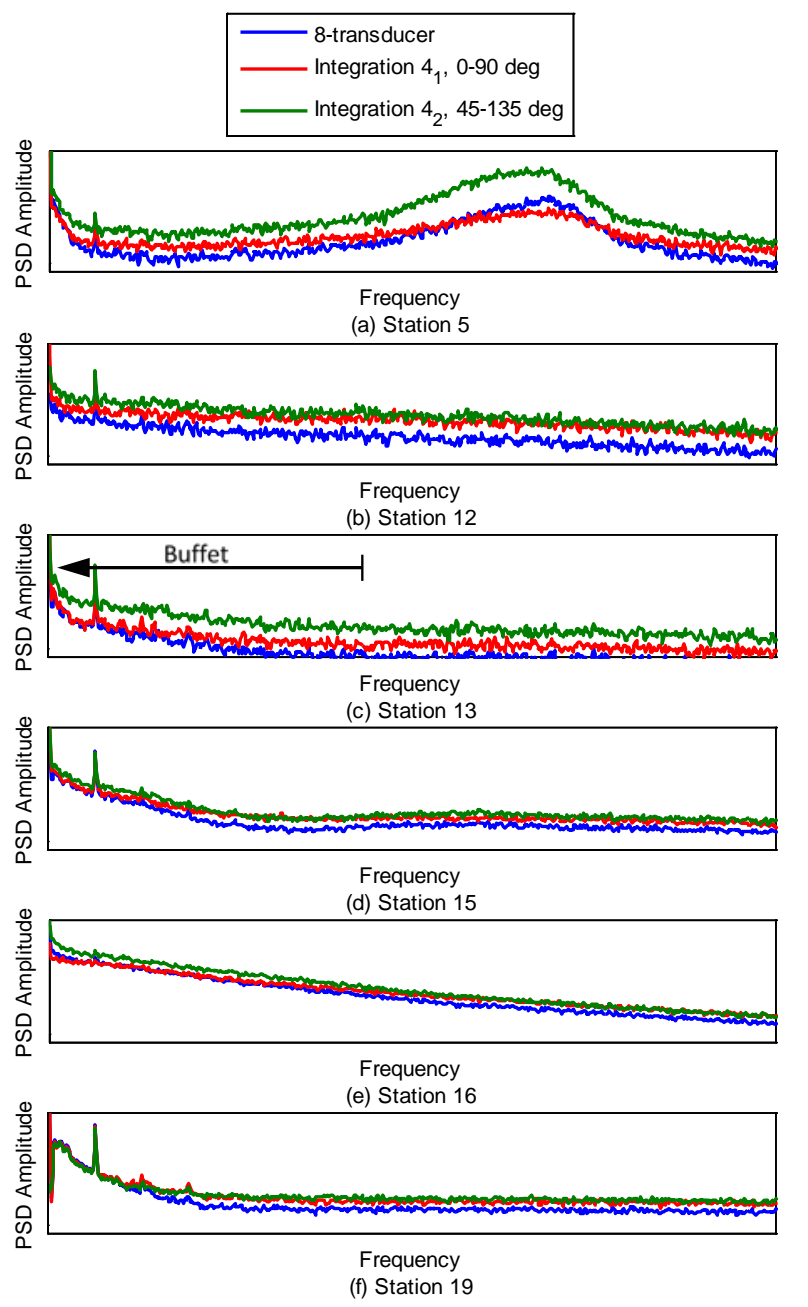

Figure 2. Comparison of PSDs for 8- and 4transducer integration BFFs, $\mathrm{M}=0.9, \alpha=0^{\circ}, \phi=0^{\circ}$.
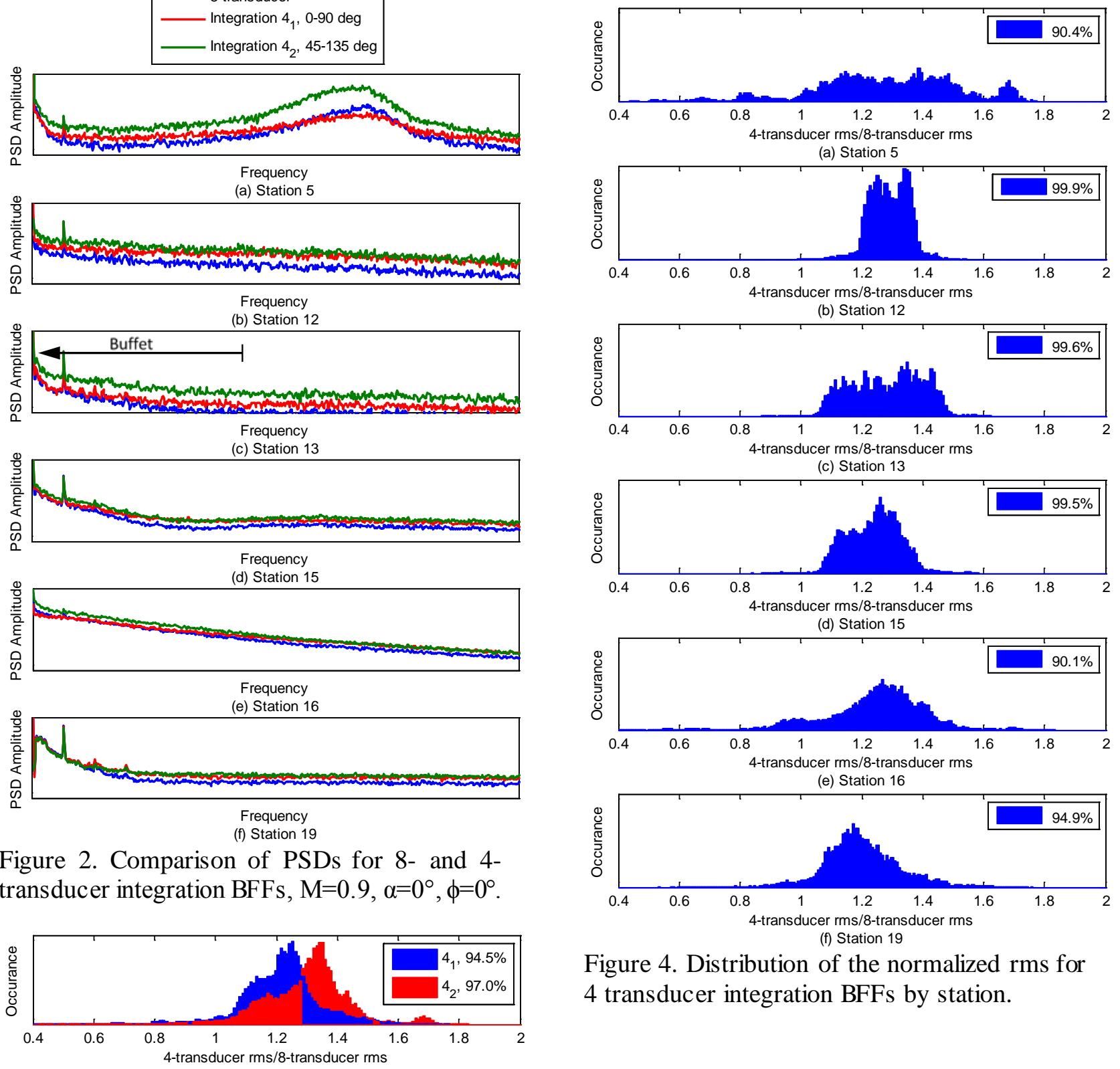

(d) Station 15

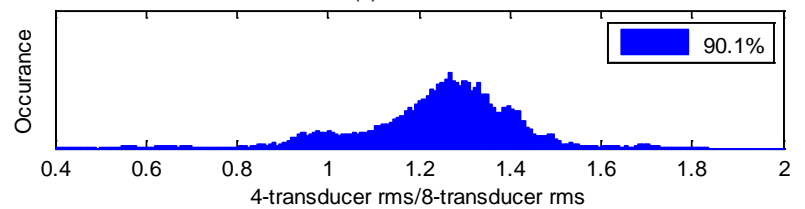
(e) Station 16

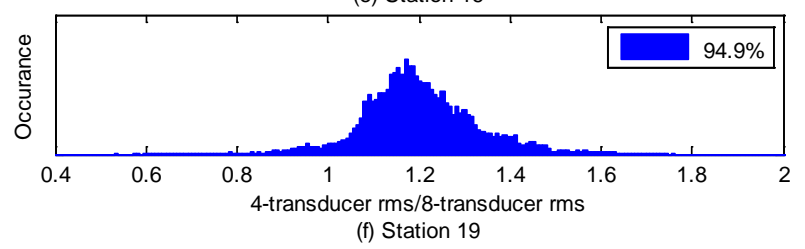

Figure 4. Distribution of the normalized rms for 4 transducer integration BFFs by station.

Figure 3. Distribution of the normalized rms for 4-transducer integration BFFs, single-body. 


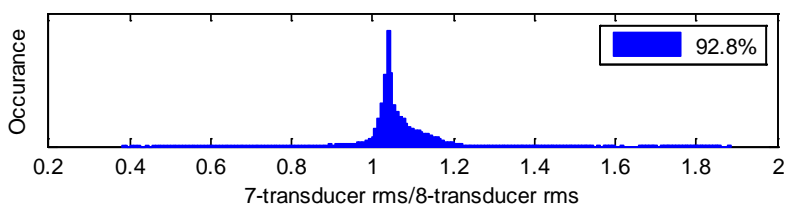

Figure 5. Distribution of the normalized rms for 7-transducer integration BFFs.

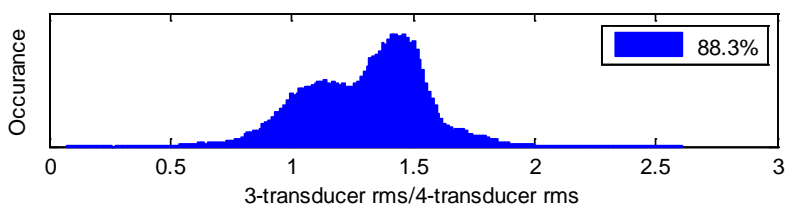

Figure 6. Distribution of the normalized rms for 3-transducer integration BFFs.

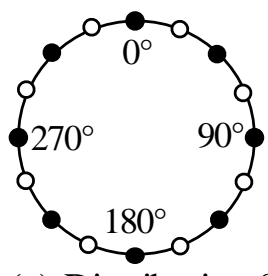

(a) Distribution 81

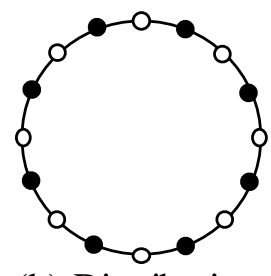

(b) Distribution 82

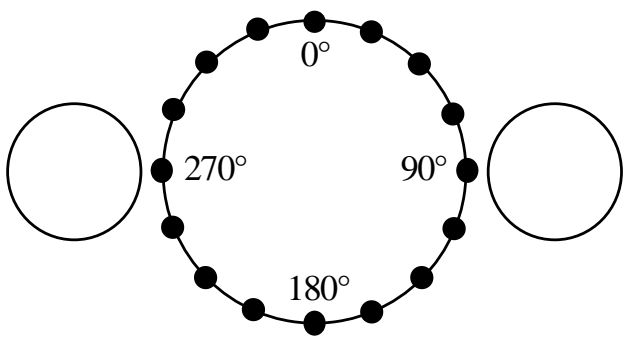

Figure 7. 16-transducer distribution.

Figure 8. Reduced axisymmetric transducer distribution for 16 -transducer ring, $\bullet=$ utilized transducers; $\bigcirc$ $=$ inactive transducers.

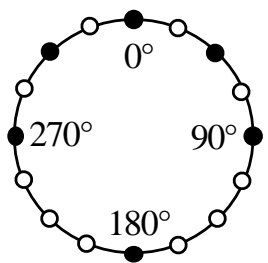

(a) Distribution 61

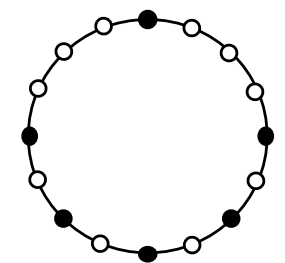

(b) Distribution 62

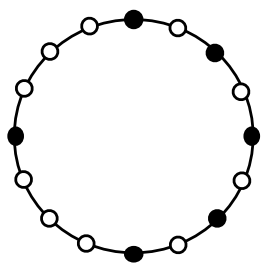

(c) Distribution 63

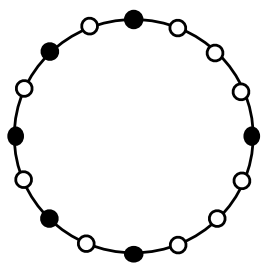

(d) Distribution 64

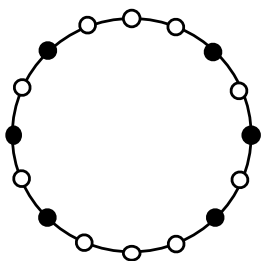

(3) Distribution 65

Figure 9. Non-axisymmetric 6-transducer distribution for 16 -transducer ring, $\bullet=$ utilized transducers; $\circ=$ inactive transducers.

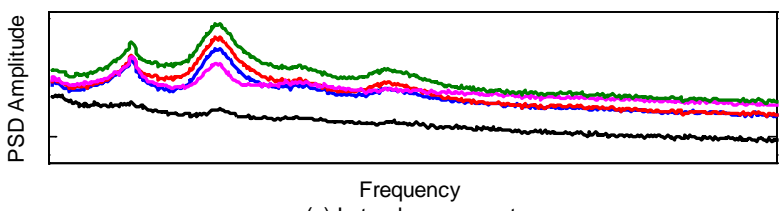

(a) Lateral component

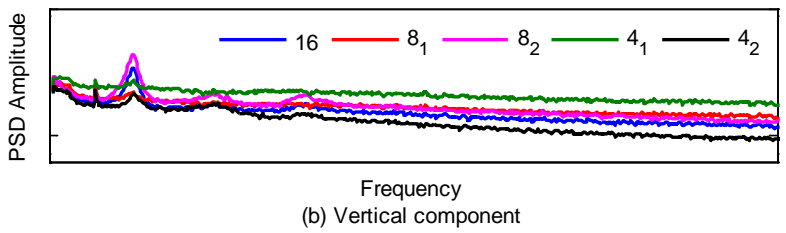

Figure 10. Effect of axisymmetric transducer density on BFF PSD, station 25, multi-body, $\mathrm{M}=0.9, \alpha=0^{\circ}, \phi=0^{\circ}$.

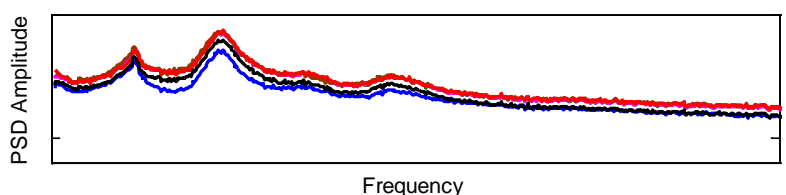

(a) Lateral component

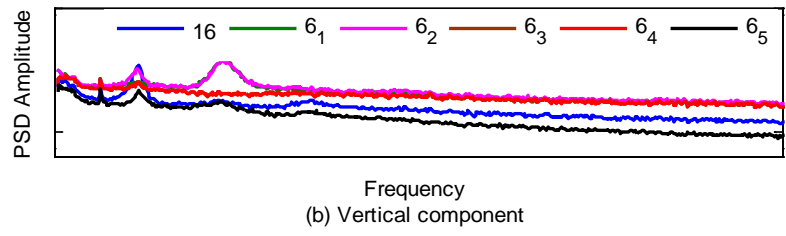

Figure 11. Effect of non-axisymmetric transducer density on BFF PSD, station 25, multi-body, $\mathrm{M}=0.9, \alpha=0^{\circ}, \phi=0^{\circ}$. 


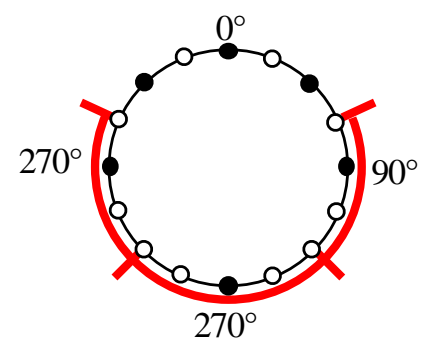

(a) Baseline

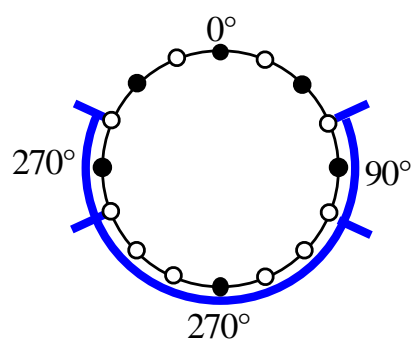

(b) Adjusted
Figure 12. Integration boundaries for nonaxisymmetric transducer distribution 6 .

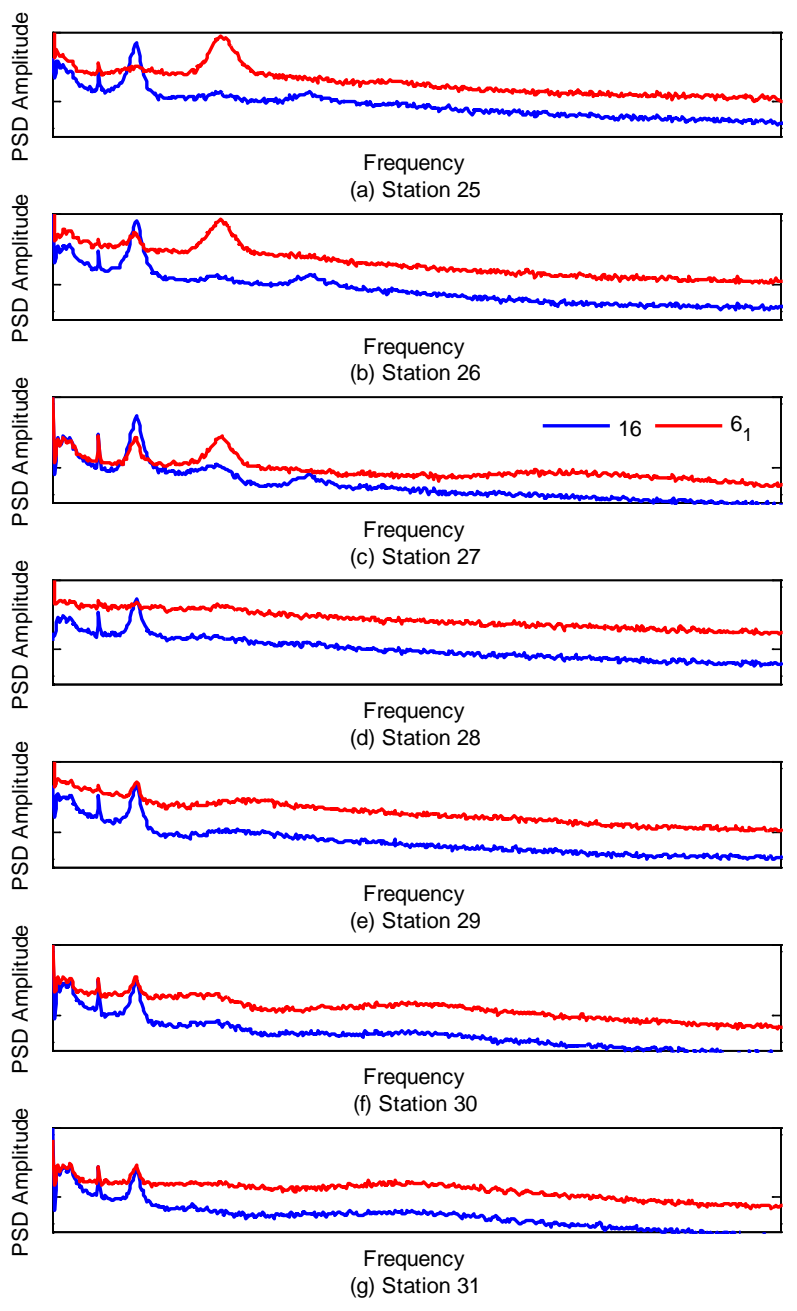

Figure 13. PSD for vertical component of BFF as a function of station, multi-body, $M=0.9, \alpha=0^{\circ}$, $\phi=0^{\circ}$.

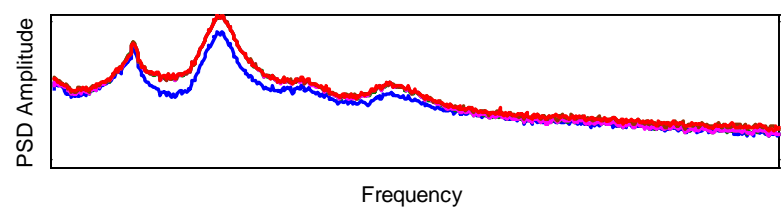

(a) Lateral component

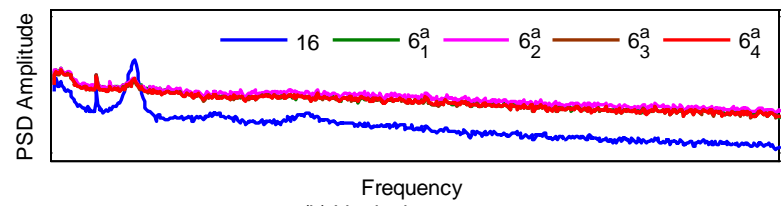

(b) Vertical component

Figure 14. Effect of integration boundary adjustment on BFF PSD for non-axisymmetric transducer density, station 25, multi-body, $\mathrm{M}=0.9, \alpha=0^{\circ}, \phi=0^{\circ}$.

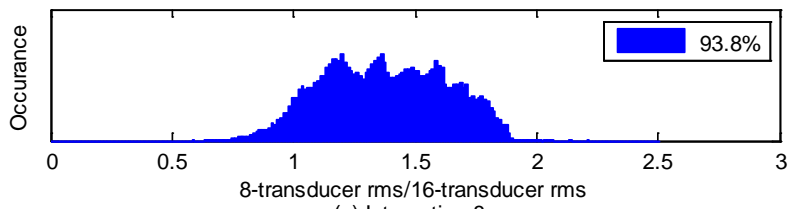
(a) Integration $8_{1}$

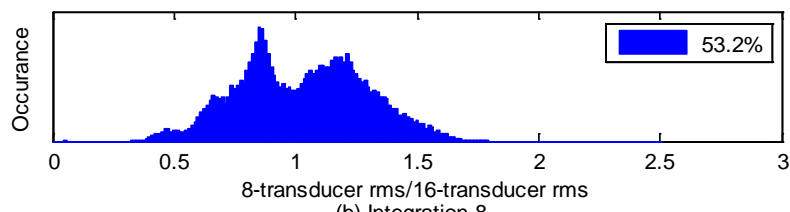
(b) Integration $8_{2}$

Figure 15. Distribution of the normalized rms for 8-transducer integration BFFs, multi-body.

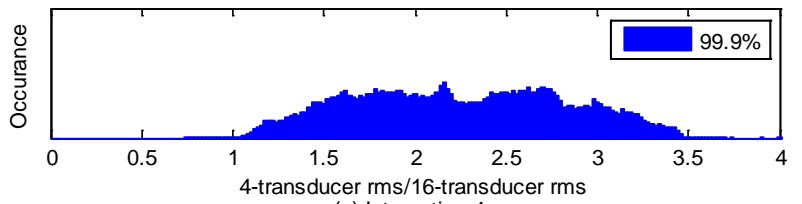

(a) Integration $4_{1}$

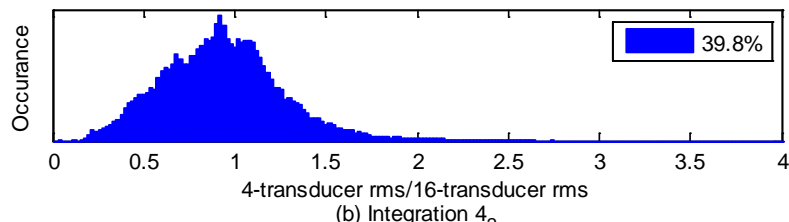

Figure 16. Distribution of the normalized rms for 4-transducer integration BFFs, multi-body. 


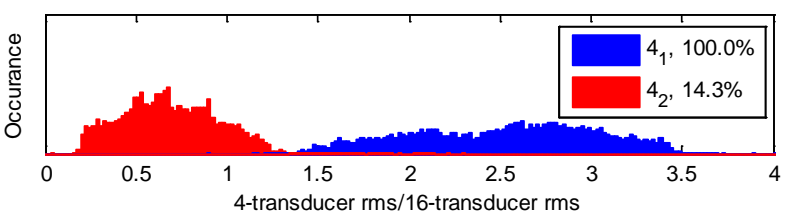

(a) Station 25

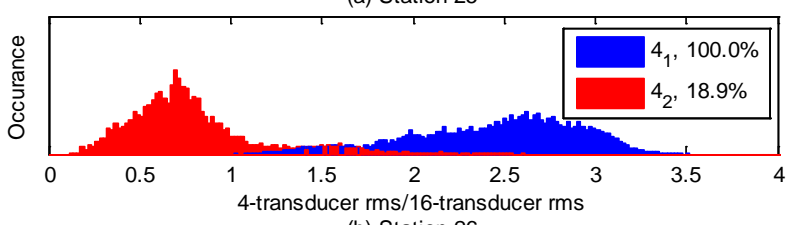

(b) Station 26

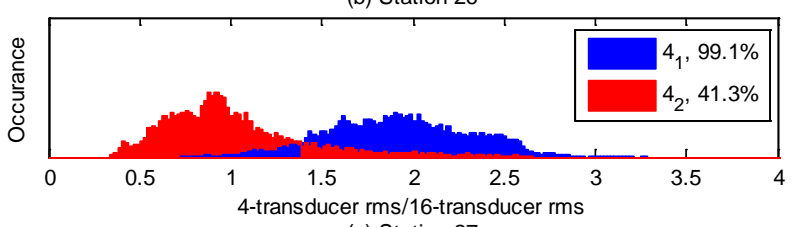

(c) Station 27

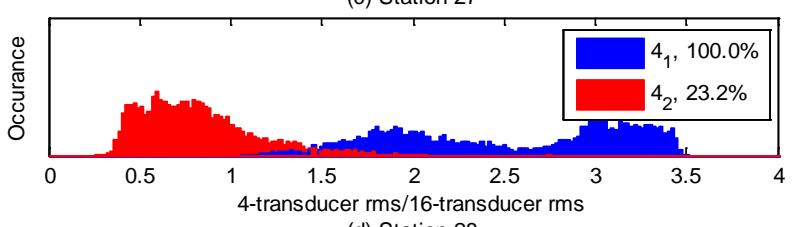

(d) Station 28

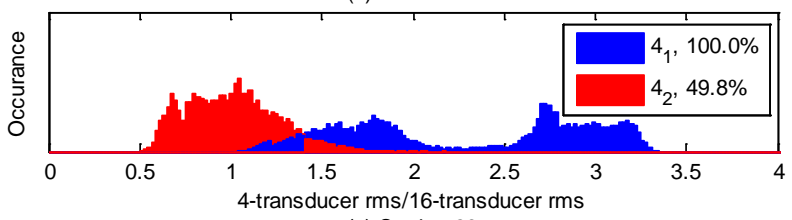

(e) Station 29

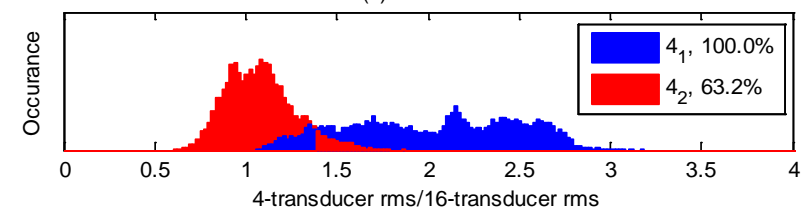

(f) Station 30

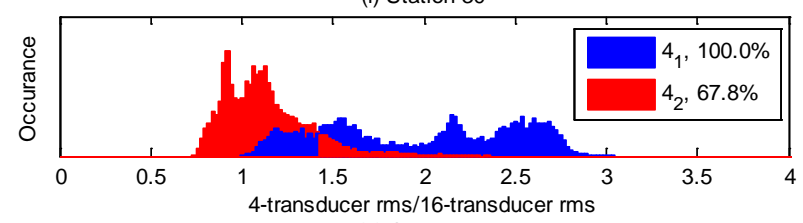

(g) Station 31

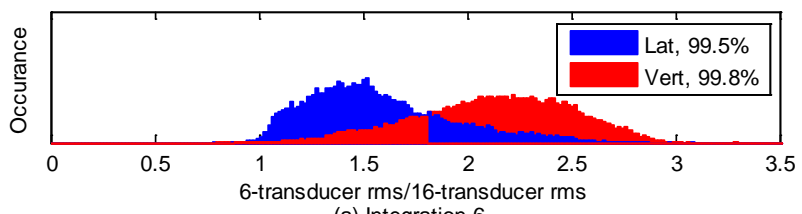

(a) Integration $6_{1}$

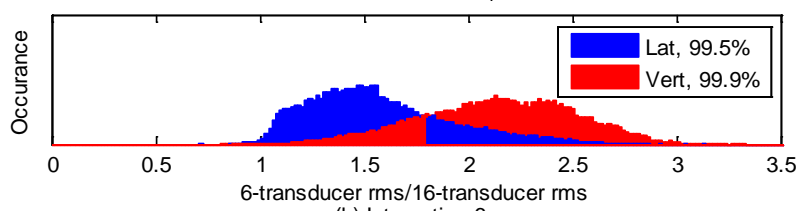

(b) Integration $6_{2}$

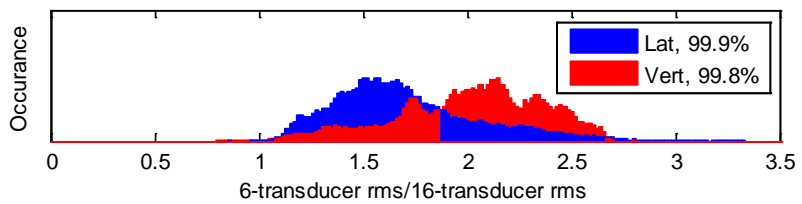

(c) Integration $6_{3}$

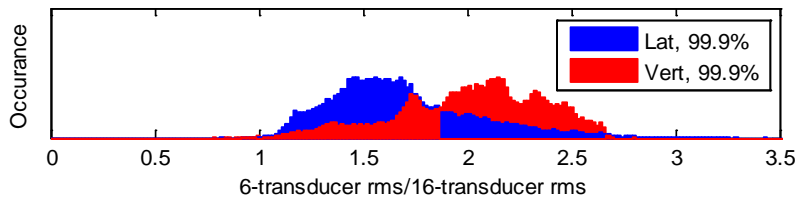

(d) Integration $6_{4}$

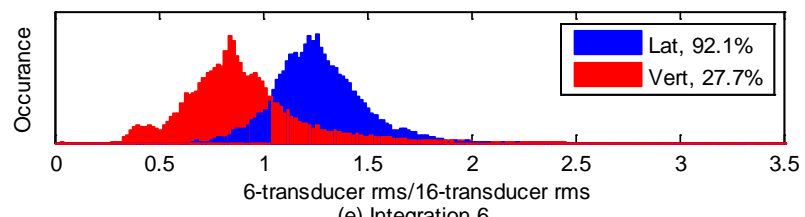

(e) Integration $6_{5}$

Figure 18. Distribution of the normalized rms for non-axisymmetric 6-transducer integration BFFs, multi-body.

Figure 17. Distribution of the normalized rms for 4-transducer integration BFFs for individual stations, multi-body. 


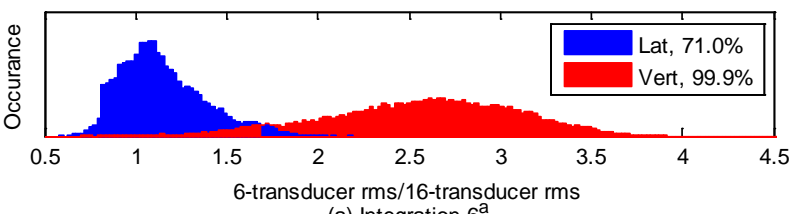

(a) Integration $6_{1}^{\mathrm{a}}$

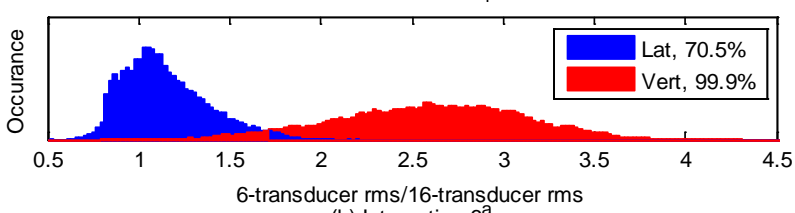

(b) Integration $6_{2}^{a}$

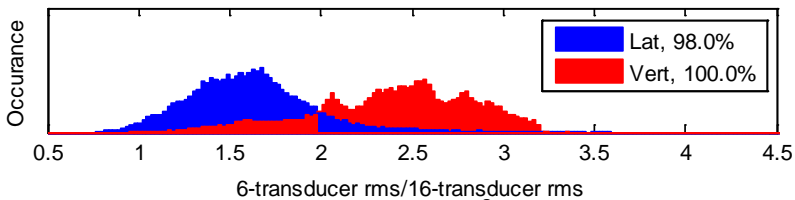

(c) Integration $6_{3}^{\mathrm{a}}$

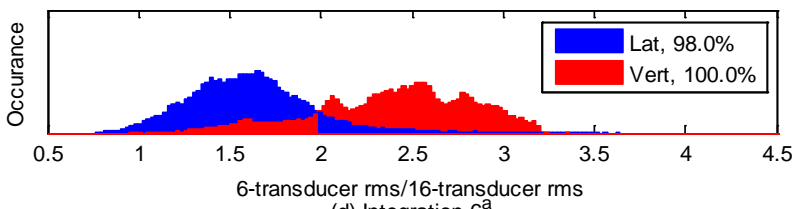

(d) Integration $6_{4}^{\mathrm{a}}$

Figure 19. Distribution of the normalized rms for non-axisymmetric 6-transducer integration BFFs, adjusted integration boundaries, multi-body.

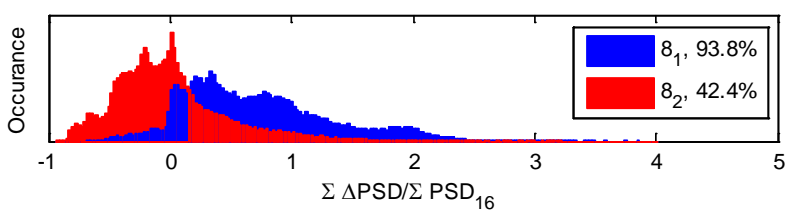

(a) Low frequency

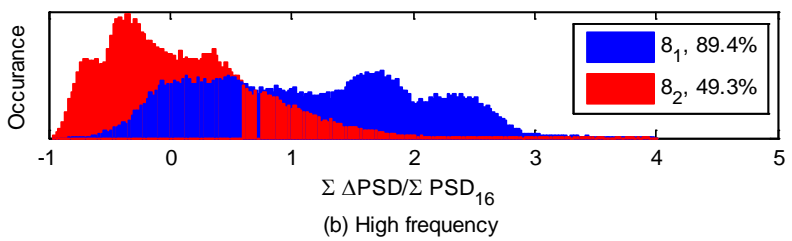

Figure 20. Normalized change in BFF energy content in the buffet frequency range, 8transducer integration, multi-body.

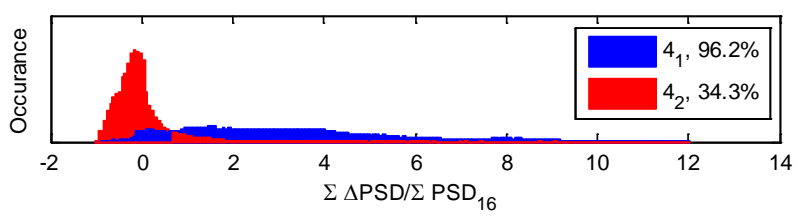

(a) Low frequency

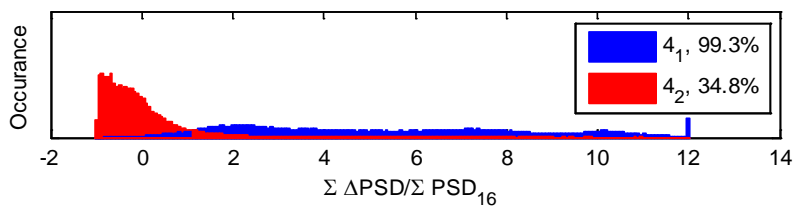

(b) High frequency

Figure 21. Normalized change in BFF energy content in the buffet frequency range, 4transducer integration, multi-body.

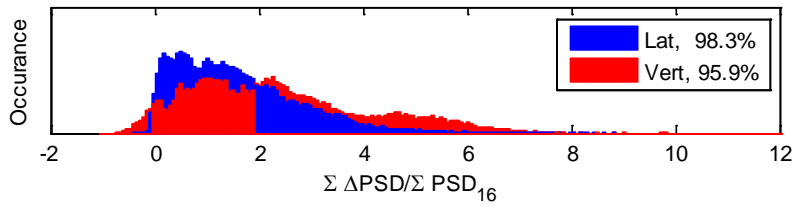

(a) Low frequency

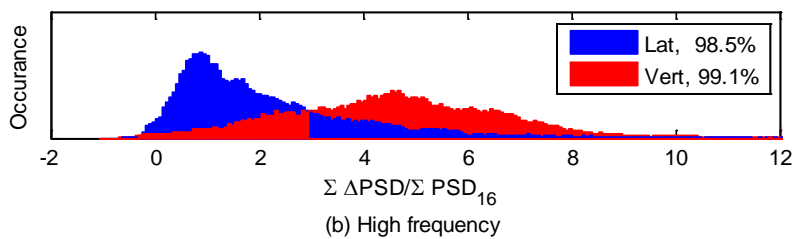

Figure 22. Normalized change in BFF energy content in the buffet frequency range, integration 61 , multi-body.

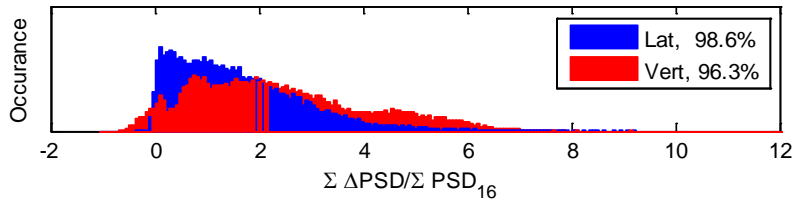

(a) Low frequency

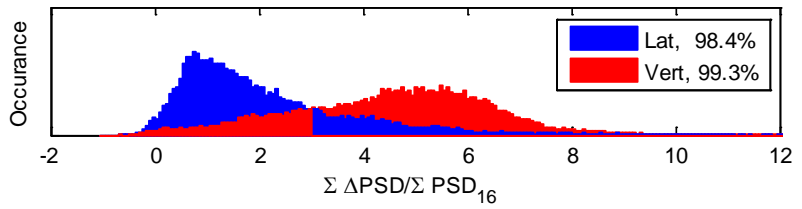

(b) High frequency

Figure 23. Normalized change in BFF energy content in the buffet frequency range, integration 62 , multi-body. 


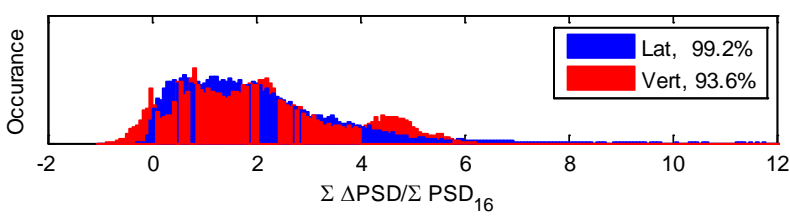

(a) Low frequency

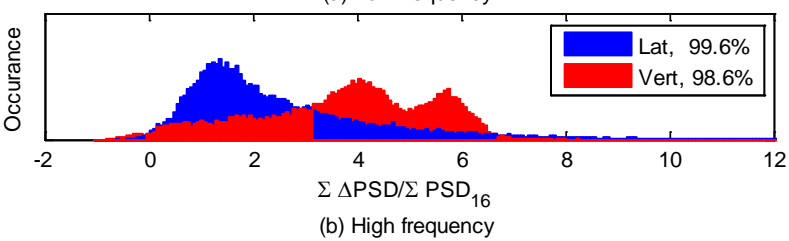

Figure 24. Normalized change in BFF energy content in the buffet frequency range, integration 63 , multi-body.

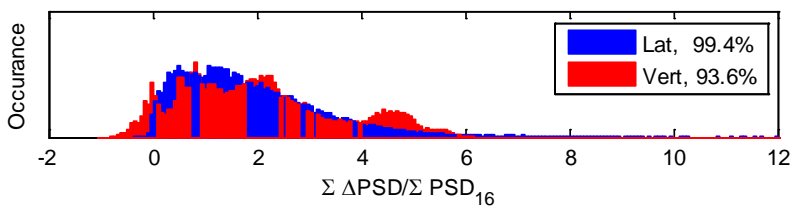

(a) Low frequency

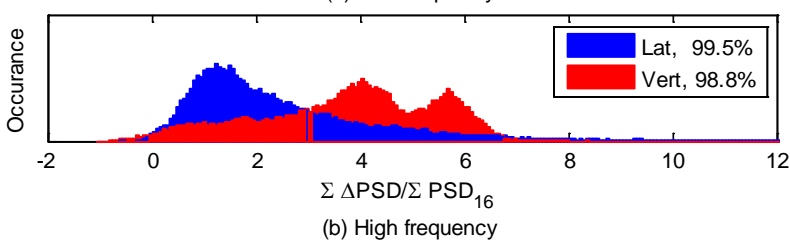

Figure 25. Normalized change in BFF energy content in the buffet frequency range, integration 64 , multi-body.

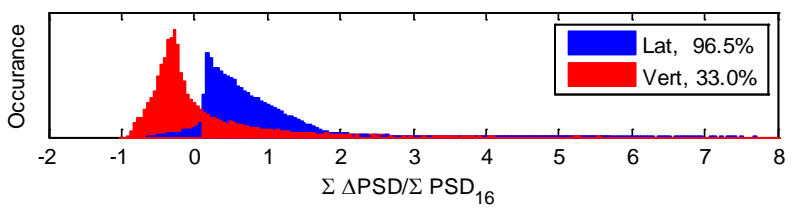

(a) Low frequency

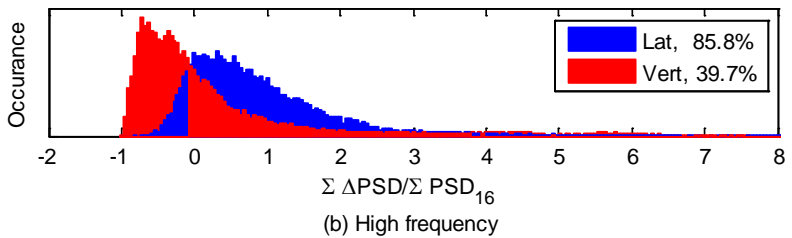

Figure 26. Normalized change in BFF energy content in the buffet frequency range, integration 65 , multi-body.

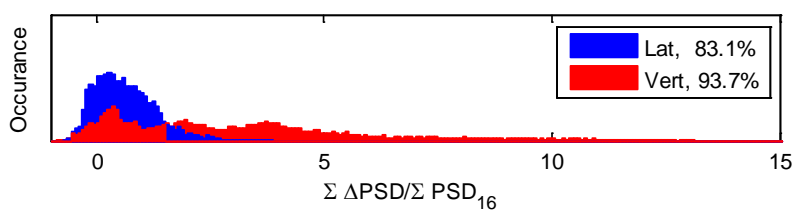

(a) Low frequency

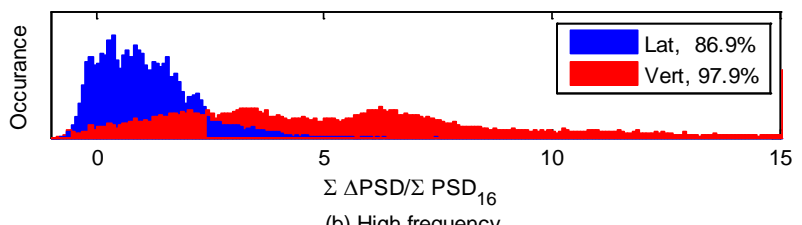

Figure 27. Normalized change in BFF energy content in the buffet frequency range, integration $61^{a}$, stations 25-27, multi-body.

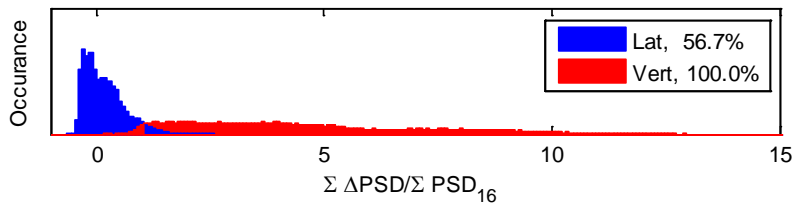

(a) Low frequency

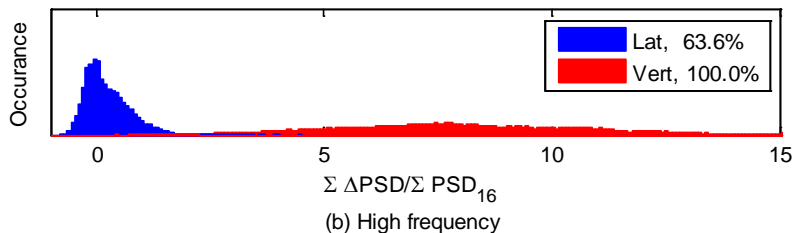

Figure 28. Normalized change in BFF energy content in the buffet frequency range, integration $61^{a}$, stations 28-31, multi-body.

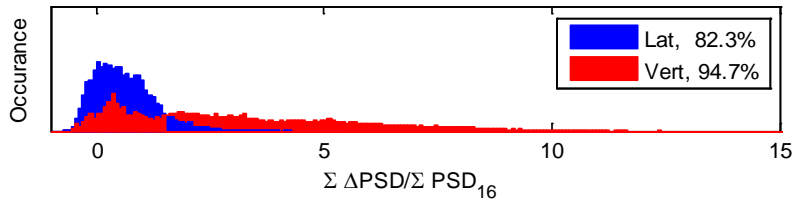

(a) Low frequency

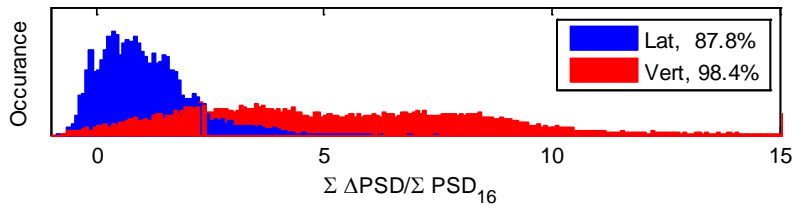

(b) High frequency

Figure 29. Normalized change in BFF energy content in the buffet frequency range, integration $62^{\mathrm{a}}$, stations 25-27, multi-body. 


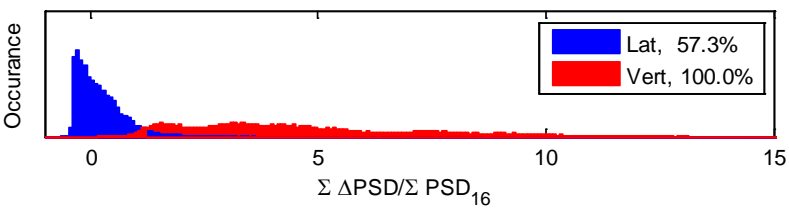

(a) Low frequency

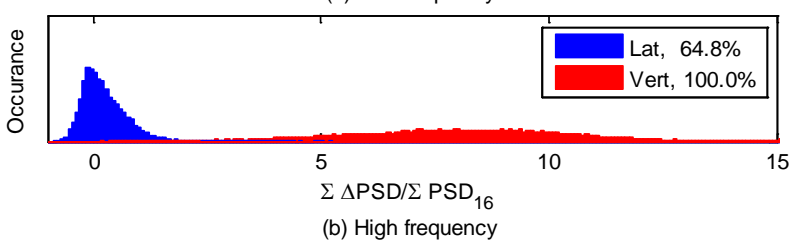

Figure 30. Normalized change in BFF energy content in the buffet frequency range, integration $6_{2}{ }^{a}$, stations 28-31, multi-body.
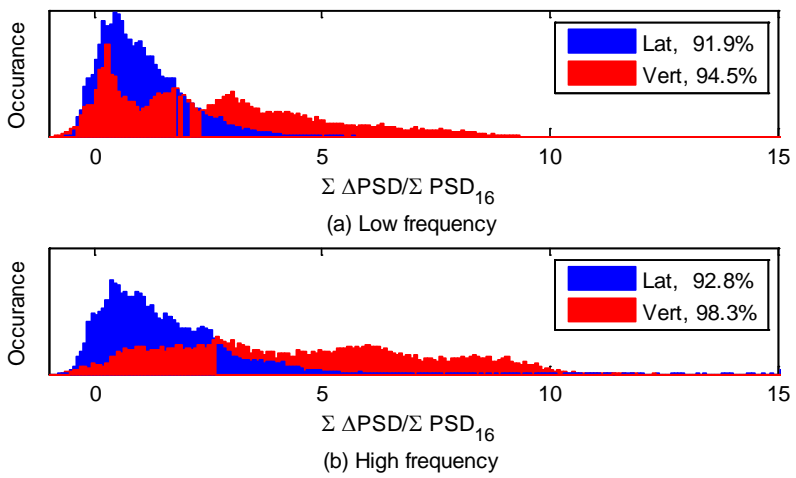

Figure 31. Normalized change in BFF energy content in the buffet frequency range, integration $63^{\text {a }}$, stations 25-27, multi-body.

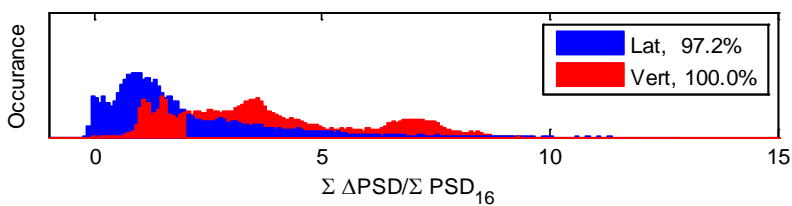

(a) Low frequency

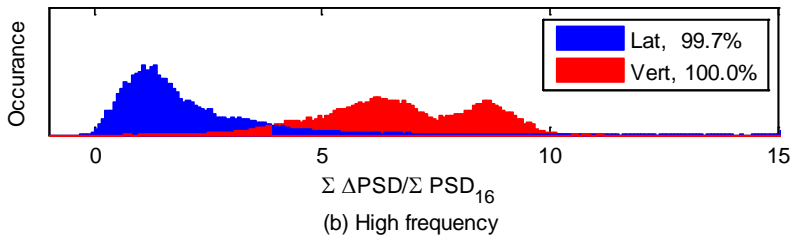

Figure 32. Normalized change in BFF energy content in the buffet frequency range, integration $63^{a}$, stations 28-31, multi-body.

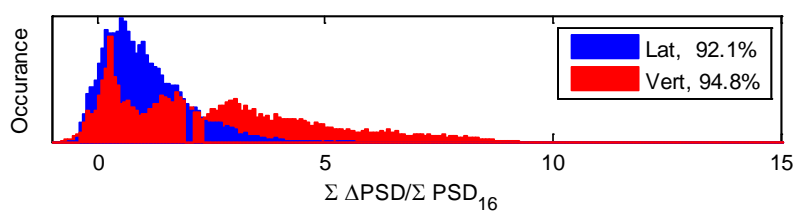

(a) Low frequency

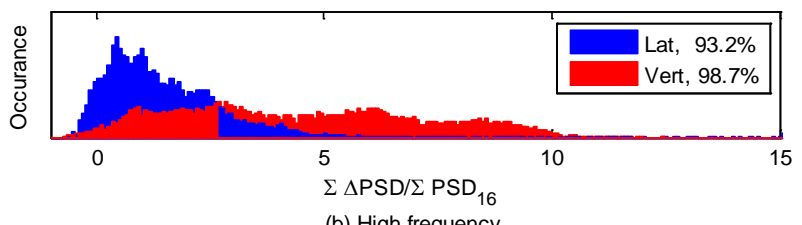

Figure 33. Normalized change in BFF energy content in the buffet frequency range, integration $64^{\text {a }}$, stations 25-27, multi-body.

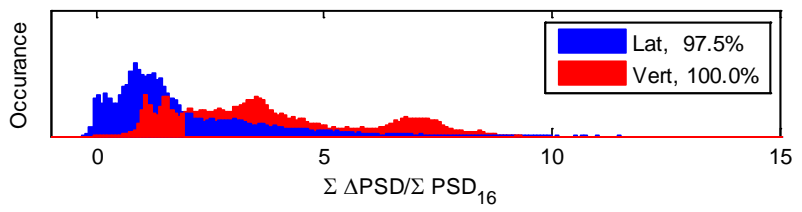

(a) Low frequency

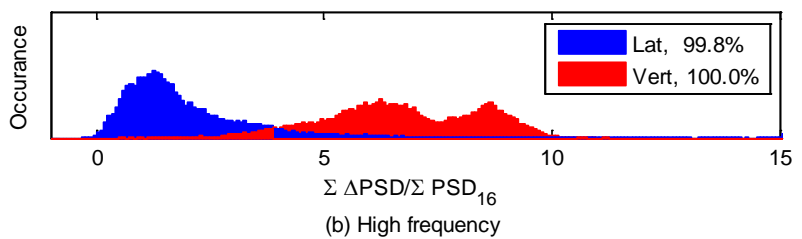

Figure 34. Normalized change in BFF energy content in the buffet frequency range, integration $64^{\text {a }}$, stations 28-31, multi-body. 